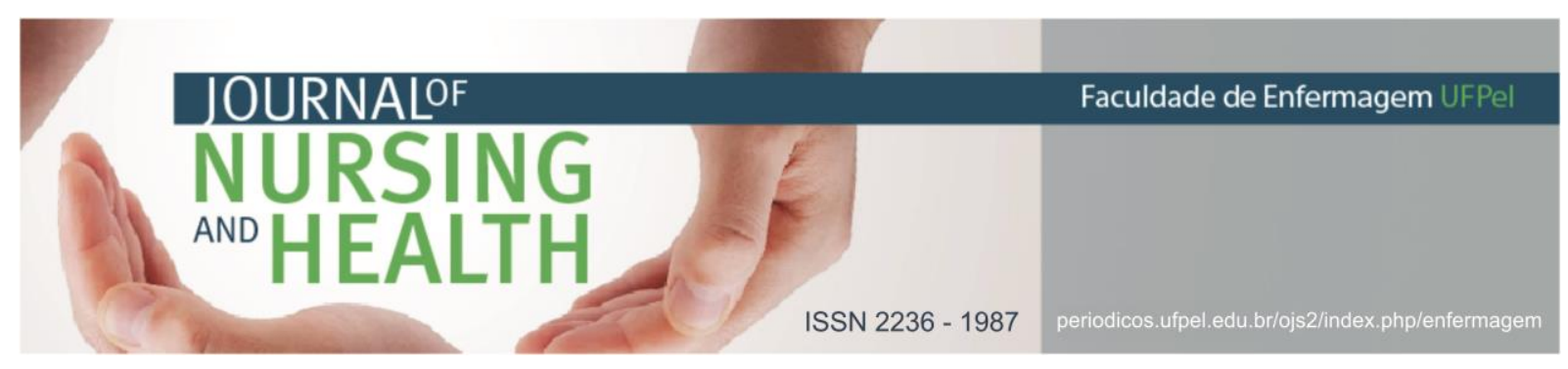

ARTÍCULO ORIGINAL

\title{
Imaginarios sociales del curso de preparación para la maternidad y la paternidad en Colombia
}

\author{
Imaginários sociais do curso de preparação para a maternidade e paternidade em \\ Colômbia
}

\section{Social imaginaries of the preparation course for maternity and paternity in Colombia}

Becerra Pabón, Ana Ceciliaํㅜ ${ }^{1}$ Vargas Escobar, Lina María²; Ardila Roa, Ilba Dorlany³

\begin{abstract}
RESUMEN
Objetivo: conocer y analizar los imaginarios sociales que fortalecen o debilitan la adhesión de las gestantes al curso de preparación para la maternidad y paternidad en un hospital público de la ciudad de Bogotá-Colombia, con el fin de generar propuestas de mejoramiento articuladas con las necesidades de las gestantes. Métodos: estudio fenomenológico, desarrollado en un hospital de la red pública de salud en Bogotá, a través de entrevistas semiestructuradas a grupos focales de gestantes inscritas al curso psicoprofiláctico en el año 2016, con análisis de datos en el programa Atlas Ti. Resultados: se clasificaron en tres categorías: importancia, razones y propuestas, en los cuales se cuenta los imaginarios de las gestantes para decidir asistir o no a los cursos psicoprofilácticos. Consideraciones finales: es importante planificar los cursos desde las necesidades educativas de las gestantes, haciéndolas protagonistas de su gestación y como sujetos con derechos sobre su cuerpo.
\end{abstract}

Descriptores: Enfermería maternoinfantil; Paternidad; Responsabilidad parental; Educación prenatal.

\section{RESUMO}

Objetivo: conhecer e analisar os imaginários sociais que fortalecem ou enfraquecem a adesão das gestantes ao curso de preparação para a maternidade e a paternidade em um hospital da rede pública da cidade de Bogotá-Colômbia, com o objetivo de gerar propostas de melhoria articuladas com as necessidades de grávidas. Métodos: estudo fenomenológico, desenvolvido em um hospital da rede pública de saúde de Bogotá, por meio de entrevistas semiestruturadas e grupos focais de gestantes inscritas no curso de psico-profilaxia, em 2016, com análise de dados no programa Atlas Ti. Resultados: as categorias foram classificados em: importância, motivos e propostas, em que o imaginário das gestantes é contado para decidir se vai ou não frequentar os cursos psico-profiláticos. Considerações finais: é importante planejar os cursos a partir das necessidades educacionais das gestantes, tornando-as protagonistas de sua gravidez e como sujeitos com direitos sobre seus corpos. Descritores: Enfermagem materno-infantil; Paternidade; Poder Familiar; Educação pré-natal.

\footnotetext{
1 Enfermera. Doctora en Ciencias de la Educación. Fundación Universitaria Juan N. Corpas, Bogotá, Colombia. E-mail: ana.becerra@juanncorpas.edu.co http://orcid.org/0000-0003-3334-1795

2 Enfermera. Doctora y Magíster en Enfermería. Universidad El Bosque, Bogotá, Colombia. E-mail: Imvargase@gmail.com http://orcid.org/0000-0003-0052-5467

${ }^{3}$ Enfermera. Magister en Ciencias de la Educación. Fundación Universitaria Juan N. Corpas, Bogotá, Colombia. E-mail: ilba.ardila@juanncorpas.edu.co http://orcid.org/0000-0003-3423-5580
} 


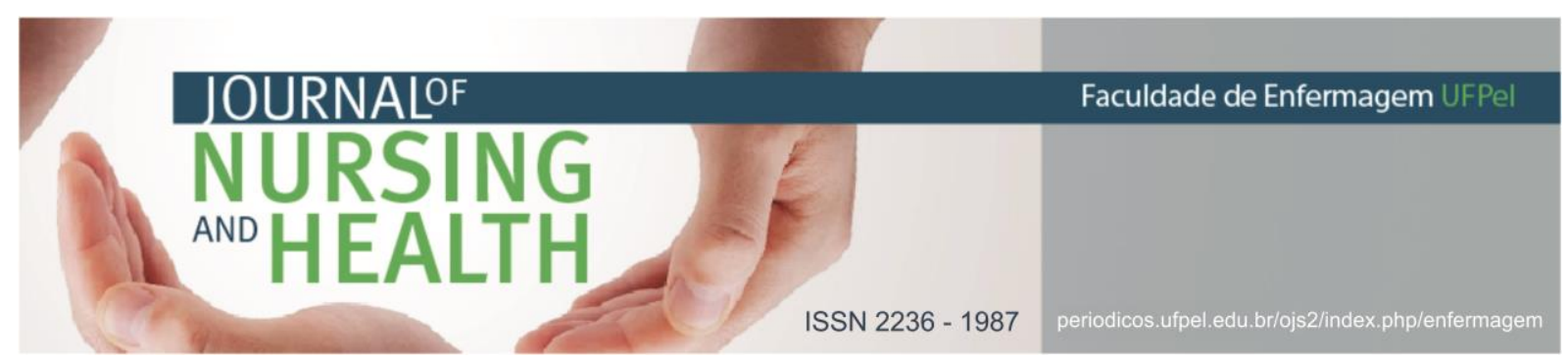

ABSTRACT

Objective: to know and analyze social imaginaries that strengthen or weaken the adhesion of pregnant women in the preparation course for maternity and paternity in a public hospital in the city of Bogotá-Colombia, in order to generate proposals for improvement articulated with the needs of pregnant. Methods: a phenomenological study, developed in a hospital of the public health network in Bogotá, through semi-structured interviews and focus groups of pregnant women enrolled in the psycho-prophylactic course in 2016, with data analysis in the Atlas Ti program. Results: the categories were classified into: importance, reasons and proposals, in which the imaginary of the pregnant women is counted to decide whether or not to attend the psycho-prophylactic courses. Final considerations: it's important to plan the courses from the educational needs of the pregnant women, making them protagonists of their pregnancy and as subjects with rights over their bodies. Descriptors: Maternal-child nursing; Paternity; Parenting; Prenatal education.

\section{INTRODUCCIÓN}

El significado de maternidad se configura a través de complejos procesos sociales y culturales y es trasmitido entre las generaciones. Las diversas trayectorias reproductivas responden a dichos procesos y generan profundas diferencias en el comportamiento sexual y reproductivo de las mujeres. ${ }^{1}$

El curso de preparación para la maternidad y la paternidad se convierte en nuestro entorno en una de las pocas oportunidades que tenemos de establecer un proceso de formación en cuidado enfocado en el proceso de gestación parto y puerperio de las mujeres gestantes y sus familias. Aun siendo conscientes de la importancia que este curso tiene para el desarrollo de la gestación, la actitud de la mujer frente al parto y la participación de la pareja y familia en el mismo, es difícil lograr un porcentaje de participación importante de las gestantes y sus familias en estas sesiones. Surgen cuestionamientos y preguntas de investigación que intentan comprender este fenómeno y acercarnos a las realidades que viven nuestras gestantes en entornos de salud tan diversos como el nuestro.
La multiplicidad de factores y creencias que las personas poseen acerca del concepto de maternidad y más aun de salud materna, es lo que Cornelius Castoradis denomina Imaginario social. Este elemento aplicado a todos los aspectos de la sociedad, podría ser la causa de éxito o fracaso de políticas de salud pública aplicadas a un determinado grupo poblacional. $^{2}$

Al realizar una búsqueda documental acerca del tema, desde contextos internacionales se encontró que los padres y madres en países como Canadá y España, al estar motivados y predispuestos a la participación activa al curso prenatal, genera efectos positivos sobre la intervención en el curso y que, frente a la asistencia a este, influyen factores como lo son el nivel de estudios, el nivel de ingresos, el tipo de trabajo y el grado de utilidad que la mujer le da a la educación prenatal. Entre las razones encontradas frente a la inasistencia a las sesiones de educación maternal, se destaca: la incompatibilidad con el horario en que se realizan las sesiones, que las mujeres no veían la necesidad de asistir a estas clases y algunas de 


\section{JOURNALOF \\ NURSING \\ MOHEALTH}

ISSN 2236 - 1987

estas recibieron comentarios de terceras personas sobre la poca utilidad de las sesiones; así mismo, se encontró que a mayores ingresos y estudios mejor es la aceptación de este programa. ${ }^{3-6}$

Por su parte en contextos latinoamericanos, se evidencian características similares en relación con las causas de la inasistencia al curso de preparación para la maternidad y paternidad. Entre estas se encontró: la calidez y respeto que percibían las gestantes por parte del personal de salud respecto a sus tradiciones culturales y/o costumbres sociales asociadas a la gestación, parto, puerperio y puericultura. ${ }^{7-8}$ Adicionalmente, se menciona que las gestantes destacan la importancia de asistir a los cursos de preparación para la maternidad y paternidad, como una oportunidad de responder dudas 0 incertidumbres que tienen frente al cuidado del bebé y de su gestación. Así mismo, se encontró como factor para la inasistencia al curso de preparación para la maternidad y paternidad, la distancia que existe entre el centro de salud $y$ sus hogares, debido que a medida que avanza su gestación se dificulta el desplazamiento hasta el centro de salud. ${ }^{9}$

En relación con el control prenatal, existe la posibilidad de encontrar conexiones entre la cultura y el sistema de salud; desde el punto de vista del profesional de la salud, se reconocen la existencia de factores culturales que dificultan la adherencia al programa prenatal en las gestantes como lo son la alimentación, los mensajes culturales que reciben en su hogar, los determinantes sociales de importancia como factores económicos y la obtención de un permiso por parte de sus jefes para ausentarse de sus actividades laborales y asistir a los encuentros programados, entre otros. Sin embargo, el profesional de salud reconoce como factores protectores la estabilidad familiar, el apoyo y participación de la pareja o la familia durante todo el proceso. ${ }^{10}$

En el contexto colombiano, las gestantes identifican que, a pesar de establecer relaciones cordiales con el personal de salud, sienten temor de contar las prácticas culturales que realizan y desean compartir sus conocimientos, ya que provienen de experiencias familiares. ${ }^{11}$ Adicionalmente, la interculturalidad y las percepciones en salud maternoperinatal que se evidencian en diferentes departamentos de Colombia, llevan a la conclusión que las diferencias culturales entre los sistemas de médicos tradicionales y facultativos, crean barreras en el cuidado de la gestante, pues, en algunas culturas las gestantes poseen conocimientos dados por el cura, curandero o "chamán"; y por el contrario en el centro médico se encuentran confundidas, ya que no tienen un adecuado respeto por sus creencias. ${ }^{10}$

En investigaciones latinoamericanas revisadas cabe destacar la importancia de la preservación de los cuidados culturales de la familia en relación a la medicina facultativa, incluyendo la necesidad de buscar el equilibrio entre lo cultural y el cuidado en salud, ofreciendo un cuidado cultural congruente y así garantizar los derechos culturales de 


\section{ISSN 2236 - 1987}

las gestantes y sus familias, previniendo las barreras para la atención intercultural que fluctúan entre las diferencias de conceptos, la poca credibilidad, el desconocimiento de las capacidades, limitaciones y experiencias negativas al intentar integrar los dos sistemas de salud. ${ }^{11-12}$

Finalmente, en la ciudad de Bogotá se encontró que algunos de los imaginarios que favorecen la adhesión al curso de preparación para la maternidad paternidad, es que la gestante tiene una visión de todo su proceso de maternidad e identifica las fortalezas que tiene la institución a donde asiste a sus controles, siendo esto un factor positivo para la caracterización de los imaginarios que se tienen frente a la dinámica del curso. ${ }^{13}$

La experiencia desde la práctica de enfermería en la realización de cursos de preparación para la maternidad y la paternidad, ha demostrado altos grados de inasistencia y desmotivación de las gestantes ante este importante proceso educativo. Es cuando surge la pregunta ¿Cuáles son los imaginarios sociales que fortalecen o debilitan la adhesión de las gestantes al curso de preparación para la maternidad y paternidad?, pregunta que se adhiere el objetivo general: "Conocer y analizar los imaginarios sociales que fortalecen o debilitan la adhesión de las gestantes al curso de preparación para la maternidad y paternidad en un hospital de la red pública de la ciudad de Bogotá-Colombia, con el fin de generar propuestas de mejoramiento articuladas con las necesidades de las gestantes".

\section{MATERIALES Y MÉTODOS}

Se aborda el problema desde el paradigma cualitativo y es la fenomenología la que permite la interpretación de los datos. Menciona que la fenomenología es entendida como la filosofía que explica la naturaleza de lo que es (serfenómenos), entendido como un paradigma explicativo pues hace uso de la ciencia para conocerla y encontrar la verdad del fenómeno ${ }^{14}$.

Se realizaron entrevistas semiestructuradas a grupos ocho grupos focales de gestantes asistentes e inasistentes al curso de preparación para la maternidad y la paternidad en un hospital de la red pública de la ciudad de Bogotá-Colombia. Se incluyeron gestantes que cursaban con una gestación de bajo riesgo. Se realizaron grupos focales heterogéneos, en donde la mitad del grupo eran inasistentes y la otra mitad eran gestantes asistentes regulares al curso. Se excluyeron las gestantes que cursaban con una gestación de alto riesgo, que no pertenecieran al sistema de afiliación de la red pública del hospital y que no quisieran participar en el estudio.

Los grupos focales fueron conformados por máximo seis gestantes, para un total de 48 gestantes entrevistadas, las cuales se contactaron telefónicamente y asistieron al hospital o Centro de Atención Médica Inmediata (CAMI) voluntariamente y sin acompañante en el momento de la entrevista. Se realizaron grabaciones de las entrevistas y se saturó la información una vez las gestantes empezaron a 


\section{JOURNALOF \\ NURSING \\ ${ }^{\text {AND }}$ HEALTH}

ISSN 2236 - 1987

de Bogotá. Los datos fueron clasificados en Unidades de Significado y/o categorías, de acuerdo a sus características. En la Figura 1 la Análisis de la Información, se especifica lo encontrado.

Las tres principales unidades de establecidos en la Resolución 00843 de $1993^{15}$ emitida por el Ministerio de Salud de Colombia, este estudio se clasificó como: "Investigación con riesgo mínimo". Sin embargo, por tratarse de mujeres gestantes, se utilizó un consentimiento informado. Todas las participantes fueron abordadas previamente a la realización del grupo focal para explicar la metodología de la investigación y se les pidió su autorización para la participación. Las participantes eran mayores de edad y estaban facultadas para otorgar su consentimiento, además tuvieron la oportunidad de elegir libremente participar en la investigación. El estudio fue aprobado por el comité de investigación de la Universidad patrocinadora del estudio, lo cual significa a su vez, aval ético del mismo.

\section{RESULTADOS Y DISCUSIÓN}

En esta investigación se realiza el análisis de la información y la emergencia de los resultados al mismo tiempo con la discusión, teniendo en cuenta que la estrategia fenomenológica permite esta triangulación. De acuerdo con el análisis realizado a los relatos de las gestantes entrevistadas, surgieron diferentes aspectos que permitieron visualizar los imaginarios de las gestantes en torno al curso de maternidad y paternidad dado en un Hospital de la red pública de la ciudad Importancia, Razones y Propuestas. La "Importancia" hace referencia a lo que la gestante consideraba importante aprender, al asistir a un curso de preparación para la maternidad y la paternidad. Las "Razones" hace referencia a las justificaciones expresadas por la gestante para no asistir al curso y las "Propuestas" son sugerencias realizadas por las gestantes para hacer el curso más estimulante y motivador.

Al analizar la primera Unidad de Significado: "Importancia" podemos observar lo relevante que es para una gestante aprender técnicas de respiración y aprender a detectar signos de alarma o complicaciones en el embarazo. También se considera muy importante para una gestante aprender a cuidar a su recién nacido ya que las gestantes refieren sentir miedo e inseguridad al momento de cargar al bebé, alimentarlo y brindarle los cuidados básicos, como lo refieren a continuación:

Me parece que es un buen curso porque hay muchas mamás y papás que son primerizos entonces no tienen ni la menor idea de cómo bañarlo, como cambiarlo, como cargarlo... entonces me parece bien (3:135). significado se denominaron: 


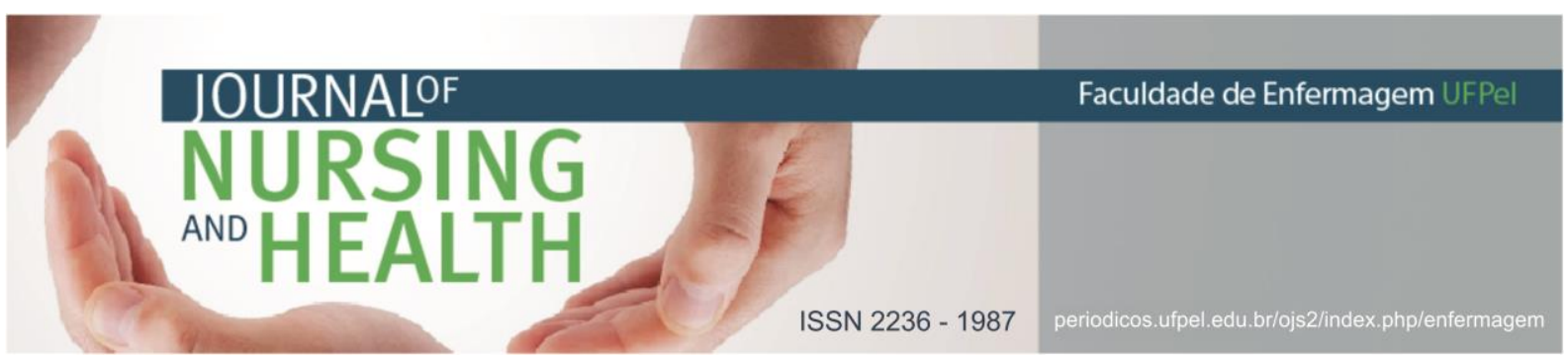

Figura 1: Mapa del análisis de la información.

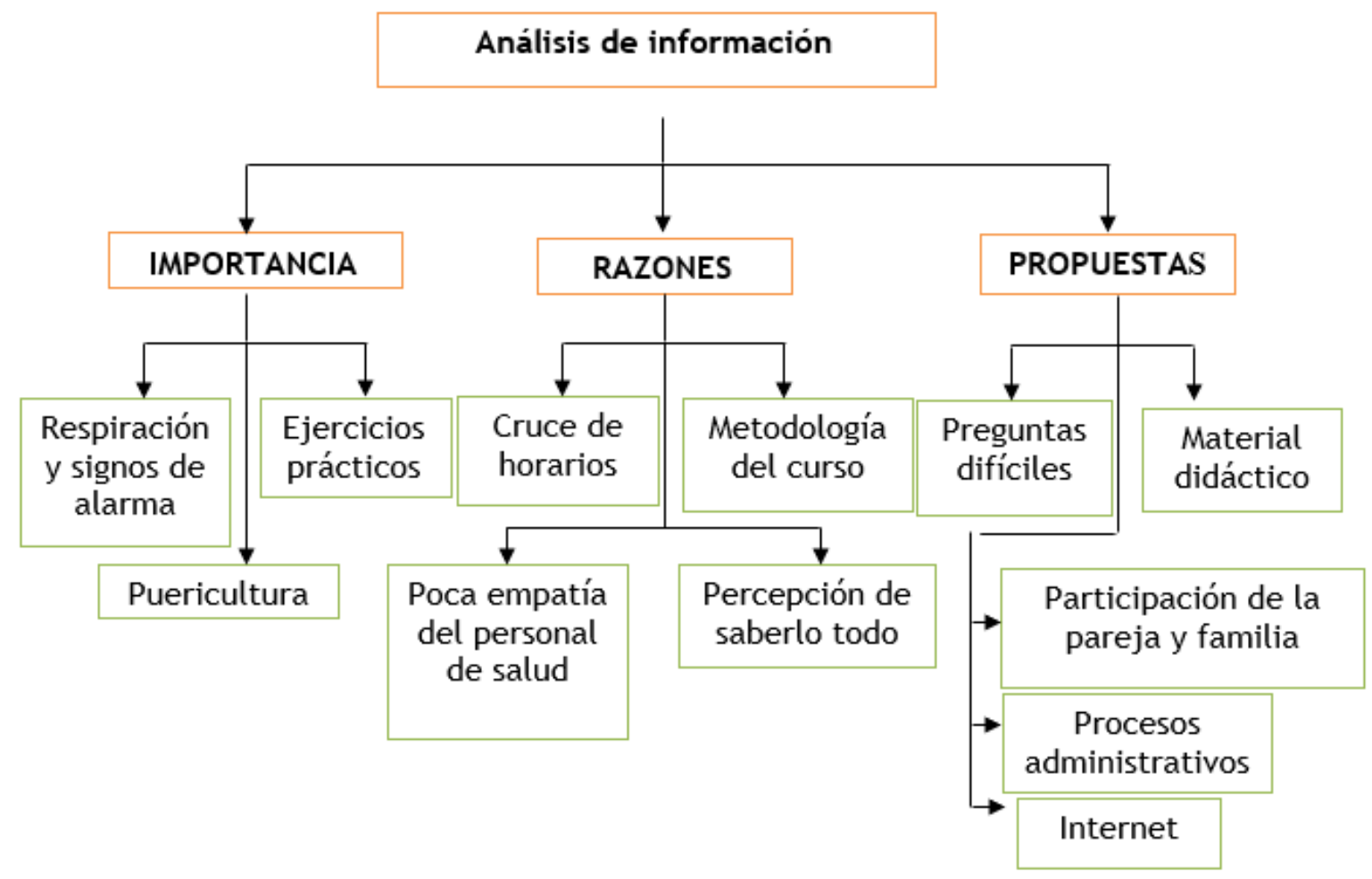

Para las gestantes entrevistadas en esta investigación es muy importante asistir al curso para hacer ejercicios, preparar y acondicionar el cuerpo para el momento del parto, disminuyendo así el riesgo para la salud materno fetal, siendo así las complicaciones durante el embarazo el mayor temor para las gestantes.

Se hace evidente en esta categoría que la gestante que asiste al curso de preparación para la maternidad y paternidad le otorga una gran importancia especialmente al aprendizaje de respiración y signos de alarma. Es decir, tal vez el temor a la complicación sea el principal motivador de asistencia de las gestantes a los cursos. También el cuidado de su bebé lo consideran

importante, incluso más importante que el cuidado de ellas mismas. Se observa nuevamente, como el miedo a la inexperiencia o la impericia en procedimientos con el recién nacido es el principal motivador de aprendizaje de las gestantes. En conclusión, asisten por temor a la complicación o a la impericia en el cuidado. No logran motivarse al aprendizaje por razones positivas, no logran ver la real importancia que tiene el curso. Teniendo en cuenta lo anterior y los resultados encontrados en el estudio denominado: Manejo del dolor durante el trabajo de parto en Gestantes de un centro de salud ${ }^{16}$, donde concluye que las mujeres que asisten a los cursos de Preparación para la maternidad y paternidad tienen una mejor preparación para la fase de trabajo de 


\section{JOURNALOF \\ NURSING \\ AND HEALTH}

ISSN 2236 - 1987

parto y adecuado manejo del dolor en relación con las que no asisten a ninguna sesión, de las cuales las mujeres que asisten a los cursos de Preparación para la maternidad y paternidad poseen los conocimientos adecuados para el manejo del dolor por medio de las técnicas de respiración y relajación que son brindados por las enfermeras que lideran los cursos.

En la segunda unidad de significado: "Razones", se encontraron varias justificaciones que la gestante da, para no asistir al curso. Dentro de las más significativas se encontraba el cruce de horarios. Las gestantes refieren que, por sus actividades diarias, el tiempo no les permite asistir al curso en el horario que propone la institución. Al respecto se encontró el siguiente relato:

Me queda muy difícil, porque estaba estudiando y trabajando, entonces no tenía tiempo (3:137).

La identificación mujernaturaleza es una constante en el pensamiento occidental. Las mujeres son conscientes de la dificultad que tendrán para llevar a cabo otras actividades no relacionadas con el rol de madre: hoy en día las mujeres tienen otros intereses al margen del rol materno, tienen objetivos profesionales y personales que con frecuencia chocan con el cumplimiento tradicional de este rol. Por este motivo hay que dotar de recursos a las mujeres y sus parejas para que puedan afrontar la conciliación de la vida familiar y laboral. ${ }^{17}$
Otra de las razones que dan las gestantes para su inasistencia hace referencia a la metodología poco motivante que tiene el curso planteado por la institución. No se cuenta en su totalidad con actividades lúdicas que permitan y faciliten el aprendizaje de las temáticas, como lo refieren a continuación:

Antes las actividades duraban una o dos horas. La vez que yo fui, en una hora y media explicaron el curso: un poquito de nutrición, planificación y ya. Luego nos acostaron en una colchoneta, hicimos ejercicios y chao. Nada, ejemplo le hablan de como respirar en el parto, cuando de pronto pasaba al siguiente tema, ellas explicaban todo en un solo tema y uno quedaba en la misma (3:108).

Es de anotar que la planeación de las sesiones no se realiza de acuerdo a las expectativas de las gestantes, sino que se ejecuta de acuerdo al documento

Preparación para la maternidad y paternidad, ${ }^{18}$ emitido por ministerio de salud. Esto hace que las sesiones educativas sean rígidas y tengan únicamente por objetivo cumplir con lo establecido previamente sin importar las dudas o necesidades educativas de las gestantes.

La falta de empatía con el personal de salud, son otras de las razones que las gestantes exponen como justificación de su inasistencia. En general manifiestan no sentirse conformes con la atención brindada por el personal de salud ya que suelen tener, en ocasiones, actitudes 


\section{JOURNALOF \\ NURSING \\ ANO HEALTH}

ISSN 2236 - 1987

mitigar todas aquellas complicaciones que se deriven en la no asistencia al curso de preparación para la maternidad y la paternidad.

Finalmente, la "percepción de saberlo todo", es otra de las razones que dan las gestantes para la inasistencia. Algunas gestantes piensan que, al haber pasado por una experiencia previa de gestación, parto y puerperio, ya no tienen nada nuevo que aprender y que no necesitan por lo tanto del curso. Al respeto se encontró el siguiente relato:

Con mi primer embarazo todo fue normal, entonces ya tenía un poquito de experiencia, entonces si yo sentía algún dolor ya sabía, no mucho, pero tenía idea y sabia manejarlo (3:149).

En este aspecto podemos observar que las gestantes no le dan la importancia que el curso merece, priorizan otro tipo de actividades domésticas sobre las actividades educativas del curso. Además, las metodologías rígidas que plantea actualmente el curso, no se basan en las necesidades de la gestante y por lo tanto no conducen a procesos de aprendizaje motivadores y estimulantes, razón por la cual se genera deserción de las gestantes en los primeros encuentros del curso. De igual manera la influencia que tiene el personal de salud en la inasistencia al curso, donde el trato y la empatía son dos herramientas fundamentales para la participación en futuras sesiones educativas por parte de las gestantes.

En la tercera y última unidad de significado encontrada: "Propuestas", surgen sugerencias, que, desde la 


\section{JOURNALOF \\ NURSING \\ M०HEALTH}

ISSN 2236 - 1987

cuidado, crianza y protección, claro está que primero deben informarse por medio de sesiones y así poder mejorar la satisfacción de la experiencia del parto, puerperio y rol como cuidadores.

Durante la gestación, las maternas comparten temores, dudas y preocupaciones sobre esta etapa. Una de ellas es acerca de la sexualidad, por este motivo refieren estar interesadas en tratar aquellos temas que son muy importantes para ellas y de los cuales existen muchos mitos, pero que no saben cómo preguntarlo o les da pena hacerlo y poder así, abrir un espacio para ello. Como se menciona en el siguiente relato:

\section{Realmente yo sí tuve esa pregunta, a mí me dijeron que cuando uno está embarazado, no se puede tener relaciones porque el hombre cuando uno está teniendo relaciones hace mucha fuerza y le puede espichar a uno la barriga; entonces va a molestar al bebé (3:36).}

Además, las gestantes entrevistadas sugieren mejorar las ayudas didácticas que se dan en los cursos, es decir, utilizar medios físicos educativos al finalizar cada sesión del curso de preparación, con el fin de obtener una mayor comprensión y asociación de la información brindada durante el mismo. El uso de internet para obtener información sobre las dudas que surgen acerca de la gestación, fue una propuesta reiterativa. La gestante en la actualidad tiene acceso a todo tipo de información y la encuentra fácilmente en la Web. La sugerencia que nos dejan 


\section{JOURNALOF \\ NURSING \\ AND HEALTH}

ISSN 2236 - 1987

relajación, respiración, visualización, masajes localizados, posiciones antálgicas, aromaterapia, sofrología, esferodinamia y musicoterapia, entre otros con la finalidad de mejorar el confort, motivación y entrenamiento de la madre.

\section{CONSIDERACIONES FINALES}

lo mira por internet, por YouTube ve toda la información del bebe" (3:151).

Está en internet, pues la verdad siempre que voy a mirar algo de bebes miro en internet (3:74).

En general las propuestas que nos dejan las gestantes hacen referencia a incentivar a la familia de la gestante a participar en el curso de preparación por medio actividades lúdicas que permitan la integración de los mismos, incluyendo temáticas de interés como la preparación para el proceso de la gestación y cuidados del recién nacido, se deben complementar temas como sexualidad durante la gestación debido a que continúan existiendo muchos mitos y temores en especial por parte del padre. Así mismo, es importante retroalimentar después de cada una de las sesiones, a través de preguntas y dudas que tengan las gestantes respecto al tema dado, debido a que las gestantes sentían que no tenían un ambiente propio para expresarlas. Por último, para las gestantes es importante realizar actividades físicas en forma activa que le permitan sentir que el curso de preparación no es solo teoría. Una investigación en Perú 21 (Profilaxis Obstétrica de Emergencia) muestra su experiencia en la utilización de métodos y técnicas básicas y complementarias como
Es necesario replantear las formas de abordar los procesos educativos de las gestantes. La población de gestantes y sus familias tienen unas particularidades específicas que debemos tener en cuenta al planificar estos cursos. Consideramos que la mujer gestante es la que debe estar en el centro de la planeación educativa y a través de sus necesidades de aprendizaje es que debemos realizar la planificación de las actividades lúdico-metodológicas encaminadas a empoderar a la gestante en su proceso de gestación.

La gestante no puede seguir siendo un sujeto pasivo, a la que constantemente se le desconocen sus derechos. Un sujeto que se le considera "menor de edad", sin poder de decisión y con la única participación como cuidadora del recién nacido y de informante de posibles complicaciones. Razón por la que la gestante no se halla involucrada en su proceso de gestación y por lo tanto no se siente motivada para asistir a los cursos. Siente en general, que ya no tiene nada nuevo que aprender, por lo tanto, al no verle la importancia, simplemente relega la actividad a último lugar de sus prioridades.

Poder ver la educación de la gestante de una forma distinta a la tradicional se convierte en un desafío 


\section{JOURNALOF \\ NURSING \\ ANO HEALTH}

ISSN 2236 - 1987

4. Galiano JMM, Rodríguez MD. Contribución de la educación maternal a la salud materno infantil. Revisión bibliográfica. Matronas prof. [Internet]. 2014 [acceso en 2018 mar 27];15(4):137-41. Disponible en: http: / / www. federacionmatronas.org/wpcontent/uploads/2018/01/revisioneducacion-maternal.pdf

5. Martínez J. Impacto del programa de educación maternal sobre la madre y el recién nacido. [tesis] Granada-España: Universidad de Granada; 2012. [acceso en 2016 ago 10] Disponible em: http: / /0-

hera.ugr.es.adrastea.ugr.es/tesisugr/ 21277709.pdf

6. Ortiz C. Influencia de la educación maternal en el embarazo, parto, puerperio y salud neonatal [trabajo de grado]. Cantabria-España: Universidad de Cantabria; 2014 [acceso en agosto de 2015]. Disponible en: http://repositorio.unican.es/xmlui/bi tstream/handle/10902/5229/OrtizFer nandezC.pdf?sequence $=1$

7. Rodríguez $F$, Jiménez $W$, Jiménez $C$, Coral A, Ramirez P, Ramos N. Efecto de las barreras de acceso sobre la assistência a citas de programa de control prenatal y desenlaces perinatales. Rev gerenc polít salud [Internet]. 2014 [acceso em 2018 mar 27]13(27):212-227. Disponible em: http://www.redalyc.org/html/545/54 533007012/

8. Martínez A, Paniagua D, Vuyk I, Riveros L, Mercado M, González A et al. Diferencias en las representaciones sociales de la atención prenatal en gestantes que asisten al Hospital de Minga Guazú, Paraguay. Revista cies_Knowledge_Survey_Report_2014. pdf 


\section{He}

desafíos [Internet]. 2015 [acceso en 2015 sep 9]; 9(1) 37-48. Disponible em: http://revistas.ut.edu.co/index.php/ desafíos/article/view/612/493

9. Vasconcelos-Moura M, Fernandes eSilva G, Santos C, Araujo-Silva VM. La calidad de asistencia de enfermería en el periodo prenatal desde la perspectiva de la mujer embarazada. Rev aquichan [Internet]. 2014 [acceso em 2016 sep 14];14(2):196-206. Disponible en: http: / /www.redalyc.org/articulo.oa?i $d=74131358006$

10. Faneite $P$, Rivera $C$, Rodríguez $F$, Amato R, Moreno S, Cangemi L. Consulta prenatal: motivos de inasistencia, ¿se justifica? Rev obstet ginecol Venez [Internet]. 2009 [acceso em 2018 mar 27];69(3):147-51.

Disponible en: http:/ / www.scielo.org.ve/scielo.php? script=sci_arttext\&pid=S0048-

$77322009000300002 \&$ lng $=e s$.

11. Toro LP. Atención prenatal: ¿tensiones o rutas de posibilidad entre la cultura y el sistema de salud? Pensam psicol. [Internet]. 2012 [acceso em 2015 sep5];10(2):123-33. Disponible en: http://www.scielo.org.co/scielo.php? script=sci_arttext\&pid=S1657-

$89612012000200011 \& \operatorname{lng}=e n \& n r m=i s o>$

12. Muñoz S, Castro E, Castro ZA, Chávez N, Ortega D. Interculturalidad y percepciones en salud maternoperinatal, Toribio Cauca 2008-2009. Rev univ ind santander. Salud [Internet].

[acceso 2018 mar 27];44(1):39-44.

Disponible en: https: //goo.gl/r771xX

13. Mayorga AP. Percepción sobre el control prenatal de las gestantes
ISSN 2236 - 1987

adolescentes en la E.S.E San Cristóbal, Bogotá. Tesis de Maestría Universidad Nacional de Colombia; [Internet]. 2012 [acceso em 2015 sep 5]. Disponible en: http://www.bdigital.unal.edu.co/114 72/1/598593.2012.pdf

14. Husserl E. La crisis de las ciencias europeas y la fenomenología trascendental. Editorial Prometeo; Buenos Aires, 2008.

15. Resolución $\mathrm{N}^{\circ} 008430$ del 4 de octubre de 1993. Normas científicas, técnicas y administrativas para la investigación en salud, Ministerio de Salud. [acesso em 2016 may18] Disponible en: https://goo.gl/GokC65

16. Campos de Aldana M. Manejo del dolor durante el trabajo de parto en Gestantes de un centro de salud. Revista cuidarte [Internet].2010 [acceso em 2017 enero 9] 1(1); 35-43. Disponible

en http://dx.doi.org/10.15649/cuidarte. v1i1.72

7. Gil Bello R. Las experiencias de las mujeres durante el embarazo a partir del análisis del discurso. Revista musas [Internet] 2016. [acceso em 2018 mayo 15]; 1(1): 63-81 Disponible en: http://revistes.ub.edu/index.php/MU SAS/article/view/vol1.num1.5/18490

18. Bejarano NL. Preparación para la maternidad y paternidad: "Un proyecto de vida". Bogotá-Colombia: Universidad Nacional de Colombia; 2004.

19. Vanguardia. [Internet] Bucaramanga: Estudio revela causas de inasistencia a controles prenatales. [acesso em 2017 enero 31] Disponible en: https://goo.gl/Al9llO 


\section{NURSING AND}

20. Ramírez H. y Rodríguez I. Beneficios del acompañamiento a la mujer por parte de su pareja durante el embarazo, el parto y el puerperio en relación con el vínculo paternofilial. Revisión bibliográfica matronas prof. [Internet] 2014. [acceso em 2017 ene 10]15(4): e1-e6. Disponible em: http://www.federacionmatronas.org/wpcontent/uploads/2018/01/revisionbeneficios-pareja.pdf

21. Morales S. Características de las gestantes que recibieron Psicoprofilaxis Obstétrica de Emergencia en el sector privado como una alternativa en su atención integral. Horiz med. [Internet]. 2015 [acceso em 2018 may 15]; 15(1): 61$66 . \quad$ Disponible em: http://www.scielo.org.pe/scielo.php? script=sci_arttext\&pid $=$ S1727558X2015000100009\&lng=es.

Agradecimientos: Sara Isabel Arévalo Cuello, Kenny David Ávila Manrique, Dayana Katherine García Coba, Lina Fernanda Pachón Ortíz, Lina Damaris Sánchez Sánchez, Angie Catherine Torres Cruz; quienes participaron como asistentes de la investigación.

Data de submissão: 15/05/2018

Data de aceite: 07/06/2018

Data de publicação: 26/06/2018 